\title{
An infinity of possible invariants for decaying homogeneous turbulence
}

\author{
J. C. Vassilicos \\ Turbulence, Mixing and Flow Control Group, Department of Aeronautics \\ Imperial College London, London, SW7 2BY, UK \\ Received (30 October 2010); Revised (28 December 2010)
}

\begin{abstract}
The von Kárman-Howarth equation implies an infinity of invariants corresponding to an infinity of different asymptotic behaviours of the double and triple velocity correlation functions at infinite separations. Given an asymptotic behaviour at infinity for which the Birkhoff-Saffman invariant is not infinite, there are either none, or only one or only two finite invariants. If there are two, one of them is the Loitsyansky invariant and the decay of large eddies cannot be self-similar. We examine the consequences of this infinity of invariants on a particular family of exact solutions of the von Kárman-Howarth equation.
\end{abstract}




\section{INTRODUCTION}

Results from recent laboratory experiments ${ }^{1}$ suggest that classes of homogeneous turbulence decay exist which are at odds with classical theory ${ }^{2}$. As the general theory of homogeneous turbulence decay is based on invariants of the von Kárman-Howarth equation ${ }^{2,3}$, these recent experiments call for a fresh study of what is true about these invariants. The present letter provides such a study in the context of decaying homogeneous isotropic turbulence. However, the assumption of isotropy could be dropped by following, for example, the method of Nie \& Tanveer $\stackrel{4}{\text {. }}$

\section{INVARIANTS OF THE VON KÁRMAN-HOWARTH EQUATION}

Starting from the von Kárman-Howarth equation for decaying homogeneous isotropic turbulence ${ }^{2} \cdot \frac{3}{2}$, we show that it is possible to derive an infinite number of different invariants corresponding to an infinite number of different conditions at infinity. This equation is

$$
\frac{\partial}{\partial t}\left(u^{\prime 2} f\right)=u^{\prime 3}\left(\frac{\partial k}{\partial r}+\frac{4 k}{r}\right)+2 \nu u^{\prime 2}\left(\frac{\partial^{2} f}{\partial r^{2}}+\frac{4}{r} \frac{\partial f}{\partial r}\right)
$$

where $u^{\prime}=u^{\prime}(t)$ is the r.m.s. of the turbulent fluctuating velocity component $u, u^{\prime 2} f(r, t) \equiv<u(x, t) u(x+r, t)>$ and $u^{\prime 3} k(r, t) \equiv<u^{2}(x, t) u(x+r, t)>$, the brackets signifying an average over realisations or over the spatial coordinate $x$ which is defined on the same axis as the velocity component $u$. Note that $r \geq 0$, that $f(0, t)=1$ and that reflection invariance implies $k(0, t)=0$. It is natural to assume that all derivatives of $f$ and $k$ with respect to $r$ are not infinite at $r=0$.

Given suitable conditions at infinity, equation (1) can be used to calculate the rate of change of $u^{\prime 2} \int_{0}^{+\infty} r^{m} \frac{\partial^{n} f}{\partial r^{n}} d r$ for an infinite range of values of $m$ and $n$. Repeated integrations by parts yield

$$
\begin{gathered}
\frac{d}{d t}\left[u^{\prime 2} \int_{0}^{+\infty} r^{m} \frac{\partial^{n} f}{\partial r^{n}} d r\right]= \\
(-1)^{n} u^{\prime 3} \int_{0}^{+\infty} d r r^{m-n-1} k(r)\left[4 T_{m-n+1}^{m}-T_{m-n}^{m}\right]+(-1)^{n} 2 \nu u^{\prime 2} \int_{0}^{+\infty} d r r^{m-n-2} f(r)\left[T_{m-n-1}^{m}-4(m-n-1) T_{m-n+1}^{m}\right]
\end{gathered}
$$

where $T_{m+p}^{m}=1$ and $T_{m-p}^{m}=m(m-1) \ldots(m-p)$ if $p$ is a positive integer, and $T_{m}^{m}=m$ (note that $m$ does not need to be an integer). These integrations by parts yield the right-hand side of (2) provided that $m>n+1, n \geq 0$, $\lim _{r \rightarrow \infty}\left(r^{m-n} k\right)=0$ and $\lim _{r \rightarrow \infty}\left(r^{m-n-1} f\right)=0$. The integral $\int_{0}^{+\infty} r^{m} \frac{\partial^{n} f}{\partial r^{n}} d r$ is finite if $\lim _{r \rightarrow \infty}\left(r^{m-n+1} f\right)=0$. We make the assumption that $f(r)$ and $k(r)$ do not oscillate at infinity.

Noting that $T_{m-n-1}^{m}-4(m-n-1) T_{m-n+1}^{m}=(1+n-m)\left[4 T_{m-n+1}^{m}-T_{m-n}^{m}\right]$ for all $n \geq 0$, (2) simplifies to

$$
\frac{d}{d t}\left[u^{\prime 2} \int_{0}^{+\infty} r^{m} \frac{\partial^{n} f}{\partial r^{n}} d r\right]=
$$




$$
(-1)^{n}\left(4 T_{m-n+1}^{m}-T_{m-n}^{m}\right)\left[u^{\prime 3} \int_{0}^{+\infty} d r r^{m-n-1} k(r)+2(1+n-m) \nu u^{\prime 2} \int_{0}^{+\infty} d r r^{m-n-2} f(r)\right] .
$$

By considering linear combinations of pairs of integrals $\int_{0}^{+\infty} r^{m} \frac{\partial^{n} f}{\partial r^{n}} d r$ and $\int_{0}^{+\infty} r^{m^{\prime}} \frac{\partial^{n^{\prime}} f}{\partial r^{n^{\prime}}} d r$ for which $m-n=m^{\prime}-n^{\prime} \equiv$ $M$, we can form an infinite number of invariants. There are two cases. One where $M \equiv m-n=4$, in which case there is no need to consider such linear combinations because $\left[4 T_{m-n+1}^{m}-T_{m-n}^{m}\right]=\left[4 T_{5}^{m}-T_{4}^{m}\right]=0$ for any integer $m \geq 4$. This case immediately yields

$$
\frac{d}{d t}\left[u^{\prime 2} \int_{0}^{+\infty} r^{4+n} \frac{\partial^{n} f}{\partial r^{n}} d r\right]=0
$$

for any integer $n \geq 0$ under the conditions $\lim _{r \rightarrow \infty}\left(r^{4} k\right)=0$ and $\lim _{r \rightarrow \infty}\left(r^{5} f\right)=0$. These conditions ensure that the quantity $u^{\prime 2} \int_{0}^{+\infty} r^{4+n} \frac{\partial^{n} f}{\partial r^{n}} d r$ is both finite and independent of time for any integer $n \geq 0$. When $n=0$, this quantity is the well-known Loitsyansky invariant ${ }^{7,3}$. Integrations by parts show that this quantity is proportional to the Loitsyansky invariant for any $n \geq 0$ because $\lim _{r \rightarrow \infty}\left(r^{5} f\right)=0$ and $f(r)$ is assumed not to oscillate at infinity.

The second case is for $M \neq 4$. In this case the following linear combinations of integrals $\int_{0}^{+\infty} r^{m} \frac{\partial^{n} f}{\partial r^{n}} d r$ and $\int_{0}^{+\infty} r^{m^{\prime}} \frac{\partial^{n^{\prime}} f}{\partial r^{n^{\prime}}} d r$ are invariant:

$$
I_{M n n^{\prime}} \equiv u^{\prime 2} \int_{0}^{+\infty} r^{M+n^{\prime}} \frac{\partial^{n^{\prime}} f(r)}{\partial r^{n^{\prime}}} d r+C_{M n n^{\prime}} u^{\prime 2} \int_{0}^{+\infty} r^{M+n} \frac{\partial^{n} f(r)}{\partial r^{n}} d r
$$

where $m-n=m^{\prime}-n^{\prime} \equiv M \neq 4, n$ and $n^{\prime}$ are non-negative integers such that $n \neq n^{\prime}$ and $C_{M n n^{\prime}}=$ $-(-1)^{n^{\prime}-n}\left[4 T_{M+1}^{M+n^{\prime}}-T_{M}^{M+n^{\prime}}\right] /\left[4 T_{M+1}^{M+n}-T_{M}^{M+n}\right]$. From (3),

$$
\frac{d}{d t} I_{M n n^{\prime}}=0
$$

under the conditions that $M>1, \lim _{r \rightarrow \infty}\left(r^{M} k\right)=0$ and $\lim _{r \rightarrow \infty}\left(r^{M-1} f\right)=0$ and that $I_{M n n^{\prime}}$ is well-defined. Hence, the von Kárman-Howarth equation admits an infinity of possible finite integral invariants depending on conditions at infinity.

Whilst $M$ does not have to be an integer, the smallest integer value of $M$ for which such invariants exist is $M=2$. The particular choice $M=2, n^{\prime}=0$ and $n=1$ recovers the Birkhoff-Saffman invariant $\underline{5}^{-6}$

$$
3 I_{210}=u^{\prime 2} \int_{0}^{+\infty}\left[3 r^{2} f(r)+r^{3} \frac{\partial f(r)}{\partial r}\right] d r
$$

The use of a single integral in this expression instead of the two integrals in equation (5) is significant because $3 r^{2} f+r^{3} \frac{\partial f}{\partial r}=\frac{\partial}{\partial r}\left(r^{3} f\right)$ leads to

$$
3 I_{210}=u^{\prime 2} \lim _{r \rightarrow \infty}\left(r^{3} f\right)
$$

showing that $I_{210}=0$ if $\lim _{r \rightarrow \infty}\left(r^{3} f\right)=0$, but also that $I_{210}$ takes a finite value if defined as in (7) rather than (5) and if $\lim _{r \rightarrow \infty}\left(r^{3} f\right)$ is finite. 
The Birkhoff-Saffman invariant (7) can be generalised into an infinite series of invariants in two steps. Firstly, for any $n \geq 1$, define

$$
I_{2 n 0}=u^{\prime 2} \int_{0}^{+\infty}\left[r^{2} f(r)+C_{2 n 0} r^{2+n} \frac{\partial^{n} f(r)}{\partial r^{n}}\right] d r
$$

for which the following iterative relation holds:

$$
I_{2(n+1) 0}=I_{2 n 0}+C_{2(n+1) 0} u^{\prime 2} \lim _{r \rightarrow \infty}\left(r^{3+n} \frac{\partial^{n} f}{\partial r^{n}}\right) .
$$

Hence, if $f(r, t) \approx a_{3}(t)(L(t) / r)^{3}$ (where $L(t)$ is a length-scale and $a_{3} L^{3} \not \equiv 0$ ) to leading order when $r \rightarrow \infty$, then the generalised Birkhoff-Saffman invariants $I_{2 n 0}$ are finite and their time-independence implies the time-independence of $a_{3} L^{3} u^{\prime 2}$ (and vice versa). As a second step, define

$$
I_{M n n^{\prime}} \equiv u^{\prime 2} \int_{0}^{+\infty}\left[r^{M+n^{\prime}} \frac{\partial^{n^{\prime}} f(r)}{\partial r^{n^{\prime}}}+C_{M n n^{\prime}} r^{M+n} \frac{\partial^{n} f(r)}{\partial r^{n}}\right] d r
$$

for any $M>1$ and any $n \neq n^{\prime}$. Noting that $C_{M n\left(n^{\prime}+1\right)}=-\left(M+n^{\prime}+1\right) C_{M n n^{\prime}}$, one can derive the iterative relation

$$
I_{M n\left(n^{\prime}+1\right)}=-\left(M+n^{\prime}+1\right) I_{M n n^{\prime}}+u^{\prime 2} \lim _{r \rightarrow \infty}\left(r^{M+n^{\prime}+1} \frac{\partial^{n^{\prime}} f}{\partial r^{n^{\prime}}}\right)
$$

Under the same assumption that $f(r, t) \approx a_{3}(t)(L(t) / r)^{3}$ to leading order when $r \rightarrow \infty$, (11) and (12) applied to $M=2$ can now be used to show that all generalised Birkhoff-Saffman invariants $I_{2 n n^{\prime}}$ are finite and their timeindependence is equivalent to the time-independence of $a_{3} L^{3} u^{\prime 2}$.

Hence, our generalised Birkhoff-Saffman invariants lead to a conclusion previously reached by Birkhoff ${ }^{5}$ and Saffman ${ }^{6}$ on the basis of the constancy of (7) alone. Namely, if $f(r) \approx a_{3}(L / r)^{3}$ as $r \rightarrow \infty$, and if $\lim _{r \rightarrow \infty}\left(r^{2} k\right)=0$, then

$$
\frac{d}{d t}\left(a_{3} L^{3} u^{\prime 2}\right)=0
$$

As for any $M>1$ but different from 2 and 4 , the case $M=3$ corresponds to a new set of integral invariants. Similarly to the $M=2$ case, we rewrite the invariants $I_{3 n 0}$ using only one integral, i.e.

$$
I_{3 n 0}=u^{\prime 2} \int_{0}^{+\infty}\left[r^{3} f(r)+C_{3 n 0} r^{3+n} \frac{\partial^{n} f(r)}{\partial r^{n}}\right] d r
$$

for $n \geq 1$, and we note that

$$
4 I_{310}=u^{\prime 2} \lim _{r \rightarrow \infty}\left(r^{4} f\right)
$$

and that

$$
I_{3(n+1) 0}=I_{3 n 0}+C_{3(n+1) 0} u^{\prime 2} \lim _{r \rightarrow \infty}\left(r^{4+n} \frac{\partial^{n} f}{\partial r^{n}}\right) .
$$

The condition $\lim _{r \rightarrow \infty}\left(r^{4} f\right)=0$ under which we established the constancy of $I_{3 n 0}$ implies $I_{310}=0$. However, if $I_{3 n 0}$ is defined as in (14) rather than (5), then it is permitted to relax this condition and assume instead that 
$f(r, t) \approx a_{4}(t)(L(t) / r)^{4}$ (where $L(t)$ is a length-scale and $\left.a_{4} L^{4} \not \equiv 0\right)$ to leading order when $r \rightarrow \infty$. In this case, and without forgetting the accompanying condition $\lim _{r \rightarrow \infty}\left(r^{3} k\right)=0, I_{3 n 0}$ is finite for all $n \geq 1$, and its invariance in time leads to

$$
\frac{d}{d t}\left(a_{4} L^{4} u^{\prime 2}\right)=0
$$

An effectively identical argument to the one given above for $I_{2 n n^{\prime}}$ shows that all integral invariants $I_{3 n n^{\prime}}$ are in fact finite and time-independent under the conditions that $f(r, t) \approx a_{4}(t)(L(t) / r)^{4}$ to leading order when $r \rightarrow \infty$ and $\lim _{r \rightarrow \infty}\left(r^{3} k\right)=0$. Their time independence is also equivalent to (17).

The cases $M>4$ are similar to the cases $M=2$ and $M=3$. In general, for any $M>1$ such that $M \neq 4$, we have

$$
(M+1) I_{M 10}=u^{\prime 2} \lim _{r \rightarrow \infty}\left(r^{M+1} f\right)
$$

and

$$
I_{M(n+1) 0}=I_{M n 0}+C_{M(n+1) 0} u^{\prime 2} \lim _{r \rightarrow \infty}\left(r^{M+1+n} \frac{\partial^{n} f}{\partial r^{n}}\right)
$$

For simplicity, we focus on $I_{M n 0}$ because the argument based on (11) and (12) which we gave for $M=2$ can be applied here to show that what holds for $I_{M n 0}$ also holds for $I_{M n n^{\prime}}$. A re-definition of $I_{M n 0}$ in terms of a single integral (11) instead of (5) allows the possibility for non-zero invariants of order $M$. Specifically, with such a redefinition, it is possible to assume $f(r, t) \approx a_{M+1}(t)(L(t) / r)^{M+1}$ (where $L(t)$ is a length-scale and $a_{M+1} L^{M+1} \not \equiv 0$ ) to leading order when $r \rightarrow \infty$. In terms of the energy spectrum $E(\kappa)$ in Fourier space, this assumption takes the form $E(\kappa) \sim a_{M+1} u^{\prime 2} L(\kappa L)^{M}$ in the limit $\kappa \rightarrow 0$ when $M>1$ because $\left.^{2} E(\kappa)=\frac{u^{\prime 2}}{\pi} \int_{0}^{\infty} d r(3 f(r)+r \partial f / \partial r) \kappa r \sin \kappa r\right)$. Under this assumption and the accompanying condition $\lim _{r \rightarrow \infty}\left(r^{M} k\right)=0, I_{M n 0}$ is finite for all $n \geq 1$ and its invariance leads to

$$
\frac{d}{d t}\left(a_{M+1} L^{M+1} u^{\prime 2}\right)=0
$$

This proves a more precise version of the principle of permanence of large eddies given in p. 113 of the 1995 book by Frisch ${ }^{8}$. Note that (20) has already been obtained by Rotta ${ }^{9}$ and Lundgren ${ }^{10}$ by direct inspection but without noticing the integral invariants (11) and therefore without the resulting systematic approach given here.

We stress that $M$ does not need to be an integer for equations (6), (18), (19) and (20) to hold. However, as Rotta 9 remarked, $E(k)$ results from an integral over a spherical shell in wavenumber space ${ }^{2}$ so that any $M<2$ would imply that the spectral tensor ${ }^{2}$ (that is the Fourier transform of the velocity correlation tensor $R_{i j} \equiv<u_{i}(\mathbf{x}) u_{j}(\mathbf{x}+\mathbf{r})>$ ) diverges as $k \rightarrow 0$. We therefore limit the remainder of this letter to $M \geq 2$. There is no a priori upper limit to $M$ as the results of this section are valid for any $M>1$. 


\section{CONSEQUENCES OF THESE INVARIANTS}

It is clear that we have an infinity of possible invariants depending on the asymptotic behaviours of $f(r, t)$ and $k(r, t)$ at infinity. Some of these invariants can also be expressed in terms of the velocity correlation tensor $R_{i j}$, specifically in terms of its trace $R_{i i}$ which is a function of only $r=|\mathbf{r}|$ because of homogeneity and isotropy. In Batchelor's book on turbulence ${ }^{2}$ one can find the identity $R_{i i}(r)=u^{\prime 2}\left(3 f+r \frac{\partial f}{\partial r}\right)$ for homogeneous isotropic turbulence. Using this identity, one obtains

$$
\int r^{M-2} R_{i i} d \mathbf{r}=4 \pi \int_{0}^{\infty} r^{M} R_{i i}(r) d r=4 \pi(M-2) u^{\prime 2} \int_{0}^{\infty} r^{M} f(r) d r+4 \pi u^{\prime 2} \lim _{r \rightarrow \infty}\left(r^{M+1} f\right) .
$$

for any $M \geq 2$.

As noted by Birkhoff ${ }^{5}$ and Saffman ${ }^{5}$, this integral equals $4 \pi u^{\prime 2} \lim _{r \rightarrow \infty}\left(r^{3} f\right)$ when $M=2$ and is finite if this limit is also finite. If this limit vanishes, then so does $\int R_{i i} d \mathbf{r}$, but in both cases $\int R_{i i} d \mathbf{r}$ is an invariant.

For any $M>2, \int r^{M-2} R_{i i} d \mathbf{r}$ diverges in the case where $\lim _{r \rightarrow \infty}\left(r^{M+1} f\right)$ is finite but equals $4 \pi(M-$ 2) $u^{\prime 2} \int_{0}^{\infty} r^{M} f(r) d r$ in the case where $\lim _{r \rightarrow \infty}\left(r^{M+1} f\right)=0$. Hence, with the exception of $M=2$ and $M=4$, $\int r^{M-2} R_{i i} d \mathbf{r}$ is not in general invariant, even though there are invariants $I_{M n n^{\prime}}$ for every value of $M \geq 2$. (The case $M=4$ corresponds to $\int r^{2} R_{i i} d \mathbf{r}$ which is, in fact, the Loitsyansky invariant in a different guise.)

We now show that, for conditions at infinity which are such that the Birkhoff-Saffman invariant is not infinite, either none or only one or only two invariants are finite. Assuming that there exists a number $M_{f} \geq 2$ for which $\lim _{r \rightarrow \infty}\left(r^{M_{f}+1} f\right)=a_{M_{f}+1} L^{M_{f}+1} \not \equiv 0$ and a number $M_{g}$ for which $\lim _{r \rightarrow \infty}\left(r^{M} k\right)=0$ for any $M$ in the interval $2 \leq M<M_{g}$ but $\lim _{r \rightarrow \infty}\left(r^{M} k\right) \neq 0$ for any $M \geq M_{g}$, then the following five possibilities present themselves for $I_{M n n^{\prime}}$ redefined in terms of a single integral (11) instead of (5).

(i) $M_{g}<M_{f}$ and $M_{g}<4$, in which case all invariants $I_{M n n^{\prime}}=0$ for $M<M_{g}$ and all $I_{M n n^{\prime}}$ for $M \geq M_{g}$ are not invariant.

(ii) $M_{g}<M_{f}$ and $M_{g} \geq 4$, in which case all invariants $I_{M n n^{\prime}}=0$ for $M<M_{g}$ except the Loitsyansky invariant which is the single non-vanishing invariant, and all $I_{M n}$ for $M \geq M_{g}$ are not invariant. In this case $u^{\prime 2} \int_{0}^{+\infty} r^{4} f(r) d r$ is the only non-vanishing invariant.

(iii) $4>M_{g} \geq M_{f}$, in which case all invariants $I_{M n n^{\prime}}=0$ for $M \leq M_{f}$ but invariant $I_{M_{f} n n^{\prime}} \neq 0$ and all integrals $I_{M n n^{\prime}}$ with $M>M_{f}$ diverge.

(iv) $M_{g} \geq 4>M_{f}$ in which case all integrals $I_{M n n^{\prime}}$ for which $M<M_{f}$ are invariant but vanish and $I_{M} n n^{\prime} \neq 0$ and is invariant.

In cases (iii) and (iv), $a_{M_{f}+1} L^{M_{f}+1} u^{\prime 2}$ is the only non-vanishing invariant and $M_{f}<4$.

(v) $M_{g} \geq M_{f} \geq 4$ in which case there are only two non-vanishing invariants when $M_{f}>4$, the Loitsyansky invariant 
and $I_{M_{f} n n^{\prime}}$, i.e. $u^{\prime 2} \int_{0}^{+\infty} r^{4} f(r) d r$ and $a_{M_{f}+1} L^{M_{f}+1} u^{\prime 2}$. When $M_{f}=4, u^{\prime 2} \int_{0}^{+\infty} r^{4} f(r) d r$ is the sole non-vanishing invariant.

All in all, depending on conditions at infinity, either no finite invariants exist, or, if such exists, then either $a_{M_{f}+1} L^{M_{f}+1} u^{\prime 2}$ is the sole finite invariant with $M_{f}<4$, or $u^{\prime 2} \int_{0}^{+\infty} r^{4} f(r) d r$ is the sole finite invariant, or $u^{\prime 2} \int_{0}^{+\infty} r^{4} f(r) d r$ and $a_{M_{f}+1} L^{M_{f}+1} u^{\prime 2}$ (with $M_{f}>4$ ) are the only two finite invariants.

We close this letter by testing this conclusion on George-type self-preserving solutions ${ }^{11}, 12,1$ of (1) because of the recent claim that it might be possible to engineer self-preserving decaying homogeneous isotropic turbulence in the wind tunne $\mathbf{l}^{\underline{1}}$. These solutions are of the form $f(r, t)=f[r / l(t)]$ and $k(r, t)=b\left(\nu, u_{0}^{\prime}, l_{0}, t-t_{0}\right) \kappa[r / l(t)]$ where $u_{0}^{\prime} \equiv u^{\prime}\left(t_{0}\right)$ and $l_{0} \equiv l\left(t_{0}\right)$. Introducing these forms into (1), one obtains the solvability conditions $\frac{d}{d t} u^{\prime 2}=-2 \alpha \nu u^{\prime 2} / l^{2}$, $\frac{d}{d t} l^{2}=c \nu$ and $b=\beta \nu /\left(u^{\prime} l\right)$ where $\alpha>0, c>0$ and $\beta$ are numerical constants. It follows that

$$
u^{\prime 2}(t)=u_{0}^{\prime 2}\left[1+\frac{c \nu}{l_{0}^{2}}\left(t-t_{0}\right)\right]^{-2 \alpha / c}
$$

and

$$
l^{2}(t)=l_{0}^{2}+c \nu\left(t-t_{0}\right)
$$

If the conditions at infinity are such that no finite invariant exists, then no obvious constraint can be imposed on the exponent $2 \alpha / c$ and the rate of turbulence decay. However, in the case where the sole finite invariant is the Loitsyansky integral, then $2 \alpha / c=5 / 2$. In the case where the sole finite invariant is $a_{M_{f}+1} L^{M_{f}+1} u^{\prime 2}$ with $2 \leq M_{f}<4$, then we can take $L(t)=l(t)$ and the self-preserving form of $f$ implies that $a_{M_{f}+1}$ must be constant in time. We therefore get $2 \alpha / c=\left(M_{f}+1\right) / 2$ which lies between $3 / 2$ and $5 / 2$.

Finally, when the conditions at infinity are such that $u^{\prime 2} \int_{0}^{+\infty} r^{4} f(r) d r$ and $a_{M_{f}+1} L^{M_{f}+1} u^{\prime 2}$ (where $M_{f}>4$ ) are both finite and invariant, then no George-type self-preserving solution of (11) is allowed because of the timeindependence of $a_{M_{f}+1}$ implied by such solutions. Noting that the contribution to $\int_{0}^{+\infty} r^{4} f(r) d r$ coming from small values of $r$ is negligible, this conclusion is valid more broadly for any form of $f(r)$ which is permissible by (1) and the incompressible Navier-Stokes equations and which conforms with self-similar decay of large eddies $\stackrel{8}{-}$, i.e. for which $f(r, t) \approx f[r / l(t)]$ if $r$ is large enough and $a_{M_{f}+1}$ is time-independent as a result. Hence, if $f(r)$ decays faster than $r^{-5}$ as $r \rightarrow \infty$ (i.e. $E(k)$ drops faster than $k^{4}$ as $k \rightarrow 0$ ), and if the asympotic behaviour of the triple velocity correlation function is such that two finite invariants exist at once (case (v) above), then the decay of the large eddies cannot be self-similar. 


\section{CONCLUSIONS}

A summary of main conclusions is in the abstract. The nature of turbulence decay depends critically on the asymptotic behaviour of the double and triple velocity correlation functions at infinite separations. There are four cases depending on whether $M_{f} / M_{g}$ is larger or smaller than 1 and whether $\min \left(M_{f}, M_{g}\right)$ is larger or smaller than 4.

When $M_{f} / M_{g}$ is larger than 1 and $\min \left(M_{f}, M_{g}\right)$ is smaller than 4 there are no finite invariants. When $M_{f} / M_{g}$ is larger than 1 but $\min \left(M_{f}, M_{g}\right)$ is larger than 4 there is only one finite invariant and this is the Loitsyansky invariant. When $M_{f} / M_{g}$ is smaller than 1 , there is either one or two finite invariants dependending on whether $\min \left(M_{f}, M_{g}\right)$ is smaller or larger than 4 . In both cases $a_{M_{f}+1} L^{M_{f}+1} u^{\prime 2}$ is finite and invariant but when $\min \left(M_{f}, M_{g}\right)$ is larger than 4, Loitsyansky's $u^{\prime 2} \int_{0}^{+\infty} r^{4} f(r) d r$ is a finite invariant too.

Self-preserving turbulence decays in accordance with (22) and (23) and the infinity of possible invariants permitted by (11) cannot determine the exponent in (22) without prior knowledge of correlations between points in the turbulence which are extremely far apart. In fact, these correlations can even be such that no conclusion whatsoever can be made on the value of the exponent in (22), and the relatively high values reported for this exponent in some wind tunnel experiments ${ }^{1}$ cannot be ruled out theoretically without prior knowledge of these correlations. The self-preserving decay which seems to have been observed in some instances of fractal-generaged homogeneous turbulence ${ }^{1}$ suggests that $M_{f}$ and $M_{g}$ cannot be such that $4<M_{f}<M_{g}$ in such instances of turbulence if it is isotropic. Research with many fundamentally different ways of generating turbulence ${ }^{1}$ needs to be carried out so as to gain some understanding of what determines conditions at infinity and whether they are all physically possible.

Acknowledgements: I am grateful to Bérengère Dubrulle, Susumu Goto, Jonathan Gustafsson, John Hinch, Tom Lundgren, David Thomson and Pedro Valente for kindly reading the first version of this letter and offering valuable comments and suggestions which have helped me improve it very significantly.

1 N. Mazellier \& J.C. Vassilicos, "Turbulence without Richardson-Kolmogorov cascade". Phys. Fluids 22, 075101 (2010).

${ }^{2}$ G.K. Batchelor, "The theory of homogeneous turbulence". (Cambridge University Press, Cambridge 1953.)

${ }^{3}$ G.K. Batchelor, "Energy decay and self-preserving correlation functions in isotropic turbulence". Q. Appl. Math. 6, 97 (1948).

${ }^{4}$ Q. Nie \& S. Tanveer, "A note on third-order structure functions in turbulence". Proc. R. Soc. Lond. A 455, 
1615-1635 (1999).

${ }^{5}$ G. Birkhoff, "Fourier synthesis of homogeneous turbulence". Commun. Pure Appl. Math. 7, 19-44 (1954).

${ }^{6}$ P.G. Saffman, "The large-scale structure of homogeneous turbulence". J. Fluid Mech. 27(3), 581-593 (1967).

7 L.G. Loitsyansky, "Some basic laws of isotropic turbulent flows". Trudy Tsentr. Aero.-Giedrodin Inst. 440, 3-23 (1939).

${ }^{8}$ U. Frisch, Turbulence: The Legacy of A.N. Kolmogorov. (Cambridge University Press, Cambridge, 1995.)

9 J.C. Rotta Turbulente Strömungen (Teubner, Stuttgart 1972)

10 T.S. Lundgren, "Kolmogorov two-thirds law by matched asymptotic expansion", Phys. Fluids 14 (2), $638-642$ (2002).

11 W.K. George, "The decay of homogeneous turbulence", Phys. Fluids A 4 (7), 1492-1509 (1992).

12 W.K. George \& H. Wang, "The exponential decay of homogeneous turbulence". Phys. Fluids 21, 025108 (2009). 